\title{
Effect of foam roller, kinesiotaping and dynamic stretching on gait parameters with induced ankle muscle fatigue
}

\author{
Hye Rim Suh ${ }^{a}$, Su-Young Lee ${ }^{b}$ \\ ${ }^{a}$ Department of Physiology, College of Medicine and Neuroscience Research Institute, Korea University, Seoul, Republic of Korea \\ ${ }^{b}$ Department of Physical Therapy, Division of Health Science, Baekseok University, Cheonan, Republic of Korea
}

\begin{abstract}
Objective: The purpose of this study was to investigate the effects of foam roller (FR) stretching, kinesiotaping (KT), and dynamic stretching (DS) on gait parameters after inducing muscle fatigue in the ankle joint.

Design: Cross-sectional study.

Methods: The subjects were thirty healthy young adults between the ages of 20 and 31 years at Baekseok University who voluntarily participated in this study. The participants were randomly assigned to either the FR group, KT group, or the DS group after inducing muscle fatigue of the ankle joint. Fatigue induction of the ankle joint muscles was performed by alternating a heel up and down exercise with the standing posture on the ground. The speed was maintained at 40 beats/minute using a metronome. Subsequently, the respective intervention was applied to each group. Gait parameters were measured before and after ankle muscle fatigue induction, and after intervention using the GAITRite system. One-way ANOVA was used to compare gait parameters among groups, while repeated measures ANOVA was used to compare gait parameters within each intervention group.
\end{abstract}

Results: The FR group increased significantly in velocity, step length, and stride length except for cadence after intervention compared to after ankle muscle fatigue induction $(p<0.01)$. Furthermore, the KT group showed significant increases in velocity, cadence, step length, and stride length after intervention, especially in cadence group $(p<0.05)$. All intervention groups showed significant increases in stride length after intervention, especially the DS group $(p<0.05)$.

Conclusions: Therefore, we suggest that KT, FR, and DS can be an effective intervention on gait parameters when the ankle joint is unstable and injured.

Key Words: Athletic tape, Gait, Muscle stretching exercises, Muscular fatigue

\section{Introduction}

Muscle fatigue refers to the general tiredness of the muscle with decreased ability to produce force. The main causes which contribute to reduction in exercise capacity due to fatigue are accumulation of lactic acid, depletion of the substrate required for muscle activity, changes in biochemical components (such as electrolytes), and confusion of the muscular coordination process. During high-intensity exercise, energy is supplied mainly through anaerobic metabolism, ensuring increased use of glucose that is derived from the degradation of muscle glycogen [1]. Most activities dur- ing sports and exercise require extremely strong muscle power and endurance. However, increased muscle fatigue has an influence on game performance or in sustaining the ability to exercise [2].

The gait process, in which the nervous system and the musculoskeletal system are used together, requires continuous and repetitive coordination [3]. Normal gait is highly correlated with the ability to balance while abnormal gait is a cause of falls [4,5]. Several muscles, including the calf muscles, are involved in gait. An abnormal contraction of the calf muscle results in instability of the ankle joint and increased postural fluctuation [6]. Therefore, postural muscu-

Received: 23 August, 2018 Revised: 13 September, 2018 Accepted: 14 September, 2018

Corresponding author: Su-Young Lee (ORCID https://orcid.org/0000-0002-1634-6829)

Department of Physical Therapy, Division of Health Science, Baekseok University, 76 Munam-ro, Dongnam-gu, Cheonan 31065 , Republic of Korea Tel: 82-41-550-2546 Fax: 82-41-550-2829 E-mail: rosei118@bu.ac.kr

(c) This is an Open-Access article distributed under the terms of the Creative Commons Attribution Non-Commercial License (http://creativecommons.org/licenses/ by-nc/4.0) which permits unrestricted non-commercial use, distribution, and reproduction in any medium, provided the original work is properly cited.

Copyright (๑ 2018 Korean Academy of Physical Therapy Rehabilitation Science 
lar fatigue, for instance in the gastrocnemius and soleus, increases difficulty in maintaining balance and results in reduced postural control [7]. Perepogu and Bangal [8] showed an increase in heel-contact speed and a reduction in ankle dorsiflexion after fatigue. Furthermore, decreases in muscle strength and balance required for gait due to fatigue may increase the risk of falls in the elderly, leading to serious problems [9].

The foam roller (FR) massage is effective in stretching the whole body, increasing range of motion (ROM), and relaxing the increased muscle tone in myofascial pain syndrome, muscle shortening, and adhesion. A previous study suggested that stretching using FR prevent muscle fatigue before exercise [10]. Kinesiotaping (KT) recovers movements of the agonist in the joints and inhibits muscle tension, thereby reducing pain and edema, and improving the function of injured muscles and joints [11]. It has also been shown to improve muscular strength and endurance [12]. Taping maintains balance as well as posture, and improves muscle function for exercise performance [13]. A positive effect was reported in previous studies that analyzed muscle activity and fatigue using electromyography measurements before and after taping [14]. Additionally, dynamic stretching (DS) is accomplished through relaxation of the antagonistic muscle with contraction of the agonist within the ROM of the limbs [15]. DS has been reported to increase exercise-related motions, such as the strength of leg extension, vertical jump height, and golf swing motion, as well as to improve flexibility and prevent injuries [16]. FRs, KT, and DS are easily accessible items. Also, each item is used to reduce fatigue and is considered to be an optimal product that is efficient in reducing fatigue, which has been confirmed indirectly based on the degree of improved walking.

Since inducing fatigue interrupts activities of daily living and reduces physical abilities, reducing fatigue within a short period of time with use of simple objects can produce a positive effect. Thus, recent studies have not yet investigated gait parameters although $\mathrm{FR}, \mathrm{KT}$, and DS have been known to be effective on reducing ankle fatigue and instability. Therefore, this study aimed to investigate the effects of FR, KT, and DS on gait parameters after inducing calf muscle fatigue in healthy normal adults.

\section{Methods}

\section{Subjects}

The subjects of this study were 30 young students (20 males, 10 females) attending Baekseok University in Cheonan, Chungcheongnam-do. Subjects were provided with sufficient explanations on the experimental procedures and had voluntary participated in this study. The exclusion criteria for the study subjects were muscle or nervous system lesions, weakness or pain in the calf muscles, ankle joint, or foot, and cognitive impairment. The subjects were randomly assigned to the experimental groups. Those who refused to participate in this study during the experimental period or could not continue participating in the study were excluded. The general characteristics of the participants are shown in the Table 1 . The present study was approved by the Institutional Review Board of Baekseok University (IRB No. BUIRB-201809-HR-009).

\section{Experimental procedures}

All subjects performed fatigue-induced tasks of the calf muscles after assessing the gait parameters using the GAITRite system (CIR Systems Inc., Sparta, NJ, USA). The induction of fatigue of the subjects was confirmed by the rating of perceived exertion (RPE) index, in which it was stopped at more than 17 points in the RPE. Each group (FR, KT, and DS) consisted of ten randomly assigned subjects. Subsequently, the gait parameters were measured. The intervention on all the subjects' calf muscles was performed corresponding with each group before the assessment of the gait parameters. Gait variables were collected from 3 repetitions, of which the mean value was used as the data value.

\section{Task of inducing ankle joint muscle fatigue}

Fatigue induction of the ankle joint muscles was performed by alternating a heel up and down exercise with the standing posture on the ground. The speed was maintained at 40 beats/minute using a metronome. The maximum exercise criteria were as follows: when the ROM was less than $50 \%$, and metronome-based speed control failed, or the fatigue inducing activity could no longer be performed. The

Table 1. General characteristics of the participants

$(\mathrm{N}=30)$

\begin{tabular}{lccc}
\hline Characteristic & $\begin{array}{c}\text { Foam } \\
\text { roller }(\mathrm{n}=10)\end{array}$ & $\begin{array}{c}\text { Kinesiotaping } \\
(\mathrm{n}=10)\end{array}$ & $\begin{array}{c}\text { Dynamic } \\
\text { stretching } \\
(\mathrm{n}=10)\end{array}$ \\
\hline Age $(\mathrm{y})$ & $23.9(2.1)$ & $23.5(1.2)$ & $23.1(1.5)$ \\
Height $(\mathrm{cm})$ & $170.4(7.7)$ & $167.8(5.4)$ & $174.8(3.1)$ \\
Weight $(\mathrm{kg})$ & $66.0(10.1)$ & $63.0(8.6)$ & $71.8(4.5)$ \\
\hline
\end{tabular}

Values are presented as mean (SD). 
subjects were encouraged to perform at least 70 cycles of ankle joint exercises. In the present study, the Borg scale, which was used as the fatigue criterion, evaluated the degree of headache, muscle pain, and chest pain during exercise. The Borg scale is an index that can easily measure muscle fatigue without any physiological measurements. Although this is a subjective measure, a person's rating of exertion may provide a fairly good estimate of the level of objective physiological factors during physical activity. The scale is as follows: 7 (extremely light), 9 (very light), 11 (moderate), 13 (moderately severe), and 15 (hard), 17 (very hard), 19 (maximal) [17].

\section{Measurement equipment and methods}

\section{GAITRite system}

GAITRite system was used to measure gait variables. GAITRite is an electronic gait board with a length of $8.3 \mathrm{~m}$ and a width of $0.89 \mathrm{~m}$, with 13,824 pressure-sensitive sensors every $1.27 \mathrm{~cm}$ connected to a personal computer. Ideally, the investigator should have been blind to which order of the treatment each subject was receiving. When the subject walks on the board, a sensor detects a footprint. The subject's footprint was collected at a sampling rate of $80 \mathrm{~Hz}$ per second. Information on collected temporal and spatial variables was processed with the GAITRite GOLD (version 3.2b) software. The participants laid down and the length was measured from the anterior superior iliac spine to the medial epicondyle of the ankle prior to the assessment for inputting into the GAITRite software. The data of the gait variables were the mean values that were obtained from three

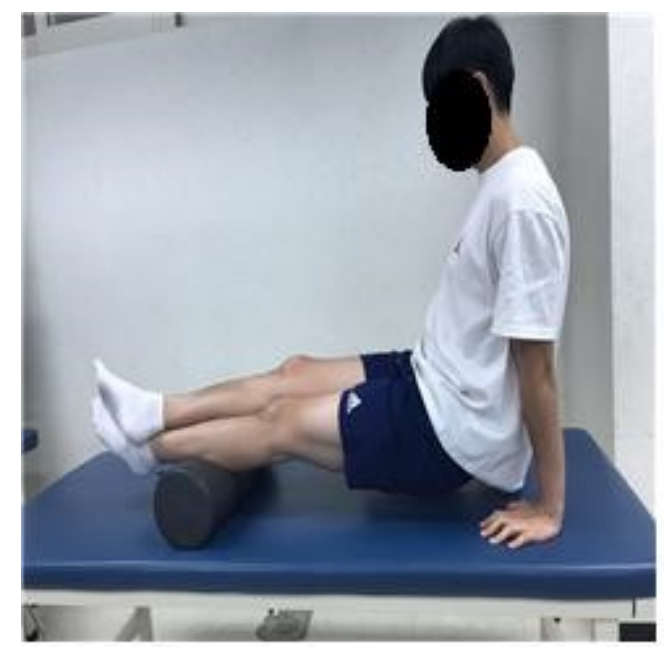

Figure 1. Foam roller intervention. repetitions. Gait speed was performed at the subject's comfortable walking speed within a distance of $10 \mathrm{~m}$. Gait variables used in this study included velocity, stride length, step length, cadence, single support, and double support time.

\section{Therapeutic intervention on ankle muscle fatigue}

\section{Foam roller massage}

The subjects in FR massage group were instructed to sit in a position with their legs extended and in a comfortable position. One leg was placed across the opposite leg to allow more pressure on the side under treatment. Subjects moved their body back and forth by supporting both arms on the floor with continual movements from the popliteal region to the Achilles tendon. The subjects were instructed to apply a large amount of pressure onto the rollers. In this way, fifteen sessions of one set were conducted, and three sets were repeated with a 1-minute break per set (Figure 1) [18].

\section{Kinesiotaping}

Kinesio Tape (2016; Nippon Sigmax Co. Ltd., Tokyo, Japan) was prepared in a Y-shape with $40 \mathrm{~cm}$ in length and $5 \mathrm{~cm}$ in width. The distal part the Y-shaped kinesio tape was attached to the heel sole with the subjects in prone position. The tape was attached separately to the medial and the lateral aspect of the calf along the calcaneus with the subjects' ankle maximally dorsiflexed. In addition, kinesio tape measuring $40 \mathrm{~cm}$ in length was attached to the popliteal fossa from calcaneus in the straight line (Figure 2) [19].

\section{Dynamic stretching}

The subjects were allowed to make an inverted V shape by

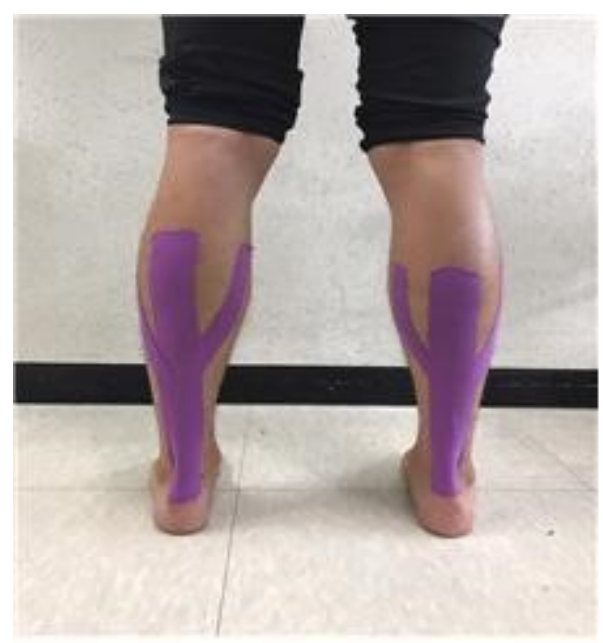

Figure 2. Kinesiotaping intervention. 
raising their hips upward from the push-up position through the extension of the elbows and knees. This posture readily stretched the calf muscles, and subjects placed their foot on the opposite ankle to enhance stretching. The subjects lifted their heels 10 to 15 times per leg as one set, with five sets and a one-minute break per set (Figure 3) [20].

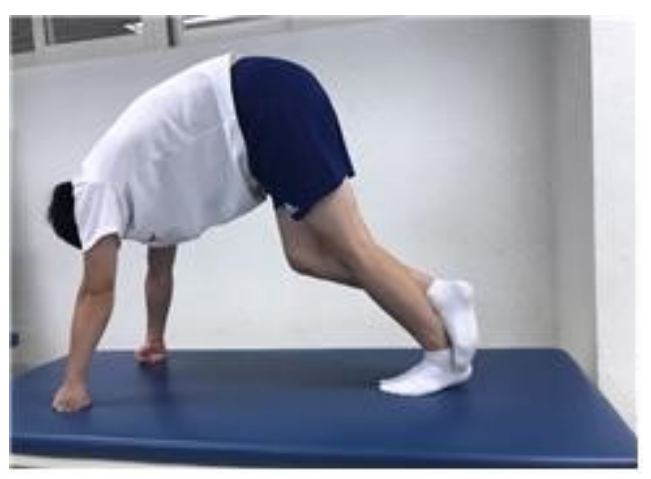

Figure 3. Dynamic stretching intervention.

\section{Statistical analysis}

This study used SPSS Statistics ver. 18.0 software (IBM Co., Armonk, NY, USA) for date analysis. The homogeneity among groups was tested using the one-way ANOVA. One-way ANOVA was used to compare the means of gait variables among groups. The Bonfferoni was used for post-hoc analysis. Repeated measures ANOVA was used to compare the means of gait variables within the groups $(p<0.05)$. The least significant difference was used for post-hoc analysis within groups. Statistical significance was set at $\alpha=0.05$.

\section{Results}

There was a significant difference in cadence, step length, and stride length among the groups after intervention $(p<0.05)$, while there was a no significant difference in other variables $(p>0.05)$. Cadence increased significantly in the

Table 2. Comparison of FR, KT, and DS on gait parameters

$(\mathrm{N}=30)$

\begin{tabular}{|c|c|c|c|c|}
\hline Variable & Pre-fatigue & Post-fatigue & After intervention & $\mathrm{F}(p)$ \\
\hline \multicolumn{5}{|l|}{ Velocity $(\mathrm{cm} / \mathrm{s})$} \\
\hline FR & $121.39(4.98)$ & $113.98(3.81)$ & $128.77(7.02)^{*}$ & $34.431(<0.01)$ \\
\hline KT & $120.95(9.77)$ & $120.41(6.07)$ & $134.34(9.09)^{*}$ & $24.448(<0.01)$ \\
\hline DS & $123.35(10.65)$ & $117.57(5.74)$ & $127.80(11.94)$ & $3.44(0.54)$ \\
\hline \multicolumn{5}{|c|}{ Cadence (step/min) } \\
\hline FR & $110.08(3.95)$ & $113.16(2.63)$ & $107.35(3.53)^{* * * *}$ & $6.188(<0.01)$ \\
\hline KT & $110.79(3.80)$ & $116.62(5.60)$ & $118.85(6.28)^{*}$ & $31.857(<0.01)$ \\
\hline DS & $110.03(3.42)$ & $114.85(5.65)$ & $108.37(3.04)^{*}$ & $6.261(<0.01)$ \\
\hline \multicolumn{5}{|l|}{ Step length $(\mathrm{cm})$} \\
\hline FR & $63.03(1.50)$ & $56.12(3.17)$ & $68.57(4.45)^{*}$ & $38.372(<0.01)$ \\
\hline KT & $66.03(3.72)$ & $56.71(2.92)$ & $69.70(2.91)^{*}$ & $63.616(<0.01)$ \\
\hline DS & $65.88(2.96)$ & $60.10(4.57)$ & $70.60(7.25)^{* * * *}$ & $3.774(0.07)$ \\
\hline \multicolumn{5}{|c|}{ Stride length $(\mathrm{cm})$} \\
\hline FR & $133.14(8.56)$ & $116.66(4.21)$ & $138.42(6.14)^{*}$ & $28.163(<0.01)$ \\
\hline KT & $130.83(7.57)$ & $124.55(9.05)$ & $136.09(5.71)^{*}$ & $14.693(<0.01)$ \\
\hline DS & $133.87(7.40)$ & $123.48(7.37)$ & $142.91(12.10)^{*}$ & $4.968(<0.05)$ \\
\hline \multicolumn{5}{|c|}{ Single limb support (\%) } \\
\hline FR & $38.44(0.74)$ & $41.03(1.91)$ & $38.46(1.75)^{*}$ & $15.488(<0.01)$ \\
\hline KT & $38.94(1.16)$ & $37.84(1.14)$ & $35.35(9.70)$ & $3.124(0.107)$ \\
\hline DS & $38.68(0.88)$ & $39.50(1.13)$ & $39.04(.85)^{* *}$ & $2.034(0.193)$ \\
\hline \multicolumn{5}{|c|}{ Double limb support (\%) } \\
\hline FR & $21.85(1.29)$ & $22.76(1.29)$ & $21.01(1.74)$ & $3.59(0.49)$ \\
\hline KT & $22.61(1.68)$ & $23.35(1.21)$ & $24.01(7.02)$ & $0.268(0.768)$ \\
\hline DS & $22.53(0.99)$ & $24.59(2.50)$ & $20.17(1.90)^{*}$ & $14.136(<0.01)$ \\
\hline
\end{tabular}

Values are presented as mean (SD).

FR: foam roller, KT: kinesiotaping, DS: dynamic stretching.

${ }^{*} p$-values show significant difference after intervention compared to post-fatigue within group in gait variables $(p<0.05)$.

** $p$-values show significant difference after intervention among groups in gait variables $(p<0.05)$. 
KT group compared to the other groups $(p<0.05)$, while step length and stride length increased significantly in the DS group $(p<0.05)$.

Velocity was not significantly different in the DS group after intervention compared to post-fatigue conditions $(p>0.05)$, while there was a significant difference in velocity in the KT and the FR groups $(p<0.05)$. Cadence was significantly increased in the KT group after intervention compared to post-fatigue conditions $(p<0.05)$. However, cadence was significantly decreased in the DS and the FR groups after intervention compared to post-fatigue conditions $(p<0.05)$. Step length was not significantly different in the DS group after intervention compared to post-fatigue conditions $(p>0.05)$, which significantly increased in the KT and FR groups after intervention compared to post-fatigue conditions $(p<0.05)$. Stride length showed a significant increase after use of a FR, KT, and DS compared to post-fatigue conditions $(p<0.05)$. Single limb support was not significantly different in the KT and DS groups after intervention compared to post-fatigue conditions $(p>0.05)$ and only the FR group was significantly decreased $(p<0.05)$. There was no significant difference in double limb support of the taping and FR groups after intervention compared to post-fatigue conditions, but it was significantly decreased in the DS group after intervention compared to post-fatigue conditions $(p<0.05)$ (Table 2).

\section{Discussion}

The purpose of this study was to investigate the effects of FR, KT, and DS interventions on gait following ankle joint fatigue in young adults. This study showed that there was a significant increase in velocity of the FR and KT groups after intervention than after fatigue, within groups. There were significant increases in cadence after intervention than after fatigue within all of the three groups, especially in the KT group. The step length showed significant increases only in the KT and the FR group after intervention than after fatigue. In addition, all of the three interventions showed significant increases in stride length within groups, especially in the DS group.

The exercise programs with stretching did not have a direct effect on balance, but the improvement of balance was sufficient [21]. KT of the dorsiflexors improved balance effectively which can be seen to have a positive effect on walking endurance and balance [22]. In addition, gait analysis has recently been shown to be a useful tool in clinical and bi- omechanical studies [23]; it provides information about abnormal exercise movement patterns and objectifies the movement mechanism. In the analysis, the time and spatial variables of gait are basically included in the evaluation, and the variables include useful information, such as speed, cadence, step length, stride length, single limb support, and double limb support. Menz et al. [24] showed that there is a significant effect on stride length and step length after exercise. Additionally, there was an interaction effect between gait, step length, and stride length according to velocity [25]. There was a significant difference in gait velocity and stride length before and after exercise; however, it showed no significant difference between cadence and double limb support, despite the significant improvement in walking speed [26]. Thus, improvements in gait variables represent a decrease in muscle fatigue.

Accelerating the gait velocity means that the stride length is longer than the increase in cadence [27]. On the other hand, decreased gait speed was reported to be associated with decreased cadence and stride length, unrelated to single limb support [28]. Additionally, the gait velocity, cadence, and stride length were significantly increased by the therapeutic intervention in a speed-dependent treadmill gait training [29]. In the case of the 16-week regular stretching exercise program, the step length and cadence increased simultaneously [30]. In a study involving children with cerebral palsy, KT application on the gluteus maximus, tibialis anterior, and quadriceps increased the childrens' gait parameters [31]. The results are similar to this study because the FR and KT interventions increased gait velocity, stride length, and step length after intervention. Furthermore, increases in all variables, including cadence was increased with KT. Gage et al. [32] reported that single limb support required concentration during gait and increased single limb support results in positive gait quality. Single limb support was the longest in the elementary school group and the shortest in the elderly group. Double limb support was the longest in the elderly group and the shortest in the elementary school group. The lower the muscle activity, the shorter the single limb support, and the longer the double limb support [33]. In our study, intervention through the FR was not effective because of the increase in single limb support in gait, but DS was effective in double limb support due to the decrease in gait.

Sustained static stretching could not be effective in single limb support because it temporarily increased posture imbalance and the risk of falling. In this study, stretching was 
not continuous, but FR was effective in the single limb support for balance. Vuillerme et al. [34] reported that inducing calf muscle fatigue in healthy college students could increase falling or injury by lowering their postural control ability. Balance refers to the ability to move or maintain weight-bearing postures, while muscle fatigue alters muscle contraction efficiency and proprioceptive information, affecting motor coordination and postural maintenance [35]. During the exercise with the FR, the patient inhales through the nose and exhales through the mouth. This can be used as an auxiliary tool for stretching and strength training, and can be seen as the most effective tool for muscle relaxation and balance enhancement. Additionally, the massage effect using FR is believed to increase tissue circulation, thereby reducing hypertension and improving recovery from fatigue [36]. Thus, FRs can be applied to reduce fatigue and improve balance.

The limitations of this study are general population. Since the subjects were of a young age, it is difficult to generalize the findings to adults of other ages or athletes. Also, we used a subjective RPE scale, despite its high reliability. It is considered that although DS increased significantly in stride length, repeated measurements weren't statistically significant. It is considered that the amount of lactic acid in the subject's blood is appropriate in exhibiting an objective physiological response. The third limitation is that we designed the group organization except control group for evidence of objective intervention. Therefore, in future studies, it is necessary to study the changes in gait in athletes and the elderly and complement the objective criteria of local fatigue and group composition.

In conclusion, this study, we investigated the effects of FR, KT, and DS on gait variables with induced ankle joint muscle fatigue. In the repeated measures within groups, the KT intervention was effective in increasing velocity, step length, stride length, and cadence for ankle fatigue, while the FR application was effective in improving all variables except for cadence. In particular, the KT was the most effective in improving cadence for ankle fatigue among groups. Additionally, all groups were effective in stride length, especially the DS group. Therefore, FR, KT, and DS with fatigue of the ankle joint can have a positive effect on gait and decrease the risk of falling.

\section{Conflict of Interest}

The authors declared no potential conflicts of interest with respect to the authorship and/or publication of this article.

\section{References}

1. Han JW, Jung DS. Changes of blood lactate and glucose variables of elite female basketball players during competition. J Nutr Sci 1997;1:69-86.

2. Go YW. The Change of catecholamine in recovery by using relaxation technic after maximal exercise. International J Human Move Sci 1996;34:472-80.

3. Jung SR, Won JI. Effects of dual-task training on balance and gait performance in patients with stroke. Phys Ther Korea 2014;21: $18-27$.

4. Verheyden G, Vereeck L, Truijen S, Troch M, Herregodts I, Lafosse $\mathrm{C}$, et al. Trunk performance after stroke and the relationship with balance, gait and functional ability. Clinical Rehabil 2006;20:451-8.

5. Ni Scanaill C, Garattini C, Greene BR, McGrath MJ. Technology innovation enabling falls risk assessment in a community. Ageing Int 2011;36:217-31.

6. Yaggie JA, Mcgregor SJ. Effects of isokinetic ankle fatigue on the maintenance of balance and postural limits. Arch Phys Med Rehbil 2002;83:224-8.

7. Roerdink M, Beek PJ. Understanding inconsistent step-length asymmetries across hemiplegic stroke patients: impairments and compensatory gait. Neurorehabil Neural Repair 2011;25:253-8.

8. Perepogu AK, Bangal PR. Preparation and characterization of free-standing pure porphyrin nanoparticles. J Chemical Sci 2008;120:485-91.

9. Cho HT. Analysis of gait and muscle activities on men with flatfoot in variety condition. Dongbang Culture University; 2011.

10. Jeong CW, Ann GY, Lee JW. Effects of pilates with foam roller on body composition, senior fitness, and hip joint ROM in rural elderly women. J Phys Growth Moto Develop 2011;19:160-70.

11. Bálint G, Szebenyi B. Non-pharmacological therapies in osteoarthritis. Baillieres Clin Rheumatol 1997;11:795-815.

12. Gilleard W, McConnell J, Parsons D. The effect of patellar taping in the onset of vastus medialis obliquus with patellofemoral pain. Phys Ther 1998;78:25-32.

13. Garrack JG. The frequency of injury mechanism of injury and epidemiology ankle sprains. Am J Sports Med 1977;5:231-42.

14. Yuk JY. The sports massage and cooling down effects on the recovery of exercise-induced hypervolume in the human lower leg after heel raising. Korea Sports Research 2005;16:451-7.

15. Murphy DR. Dynamic range of motion training: an alternative to static stretching. Chiropractic Sports Medicine 1994;8:59-66.

16. Van Erck A, Vanden Bossche L, Witvrouw E, Van der Kelen V, Wojtowicz I, Adriaenssen J, et al. Effect of whole body vibration on intracompartmental pressure in the lower leg. J Orthop Sci 2009; 14:618-22.

17. Borg G. Perceived exertion as an indicator of somatic stress. Scand J Rehabil Med 1970;2:92-8.

18. Canadian Running. The manual of Breaking down foam rolling and self-massage. Canada: Canadian Running; 2013.

19. Cho IH. The effects of compression bandage therapy and taping therapy applied to physical therapists with lower limbs edema 
and pain [Master thesis]. Daegu: Daegu University; 2012.

20. Meltzer R. The stretches you need to prevent running injuries. Fitness \& Exercise, Injury Prevention, 2015.

21. Means KM, Rodell DE, O'Sullivan PS, Cranford LA. Use of an obstacle course to assess balance and mobility in the elderly. Am J Phys Med Rehabil 1996;77:1030-6.

22. Song MH, Jeon BS, Jang JH, Cho CO. Effect of kinesio taping application on balance and gait ability in hemiplegic disables after cerebral stroke. KOSAPE 2008;16:143-59.

23. Feipel V, De Mesmaeker T, Klein P, Rooze M. Three-dimensional kinematics of the lumbar spine during treadmill walking at different speeds. Eur Spine J 2001;10:16-22.

24. Menz HB, Latt MD, Tiedemann A, Mun San Kwan M, Lord SR. Reliability of the GAITRite walkway system for the quantification of temporo-spatial parameters of gait in young and older people. Gait Posture 2004;20:20-5.

25. Lee GS. Analysis of temporal and spatial gait parameters of male and female in 20s and 60s. Korean Phys Edu Assoc For Women 2007;21:55-66.

26. Park SH. Comparative analysis of physical fitness, gait parameter, and lower extremity muscle activity in stroke patients by exercise types [Master thesis]. Changwon: Changwon University; 2008.

27. Whittle MW. Gait analysis: an introduction. 4th ed. New York: Butterworth-Heinemann; 2007.

28. Beauchet O, Dubost V, Aminian K, Gonthier R, Kressig RW.
Dual-task-related gait changes in the elderly: does the type of cognitive task matter? J Mot Behav 2005;37:259-64.

29. Pohl PS, Duncan PW, Perera S, Liu W, Lai SM, Studenski S, et al. Influence of stroke-related impairments on performance in 6-minute walk test. J Rehabil Res Dev 2002;39:439-44.

30. Lee JC, Choi SO, Yoon SJ, Kang KH, Bae JJ. Effect of stretching exercise on the proprioceptive and gait ability in elderly women. J Sport and Leisure Studies 2010;39:575-86.

31. Kim MK, Cha HG. The effects of ankle joint taping on gait and balance ability of healthy adults. J Phys Ther Sci 2015;27: 2913-4.

32. Gage WH, Sleik RJ, Polych MA, McKenzie NC, Brown LA. The allocation of attention during locomotion is altered by anxiety. Exp Brain Res 2003;150:385-94.

33. Chae JB, Gong SH, Kim DJ, Kim RJ, Kim TY, Lee SH. Research for temporal-spatial parameter of the gait according to age. PNF\&Mov 2008:6:19-30.

34. Vuillerme N, Forestier N, Nougier V. Attentional demands and postural sway: the effect of the calf muscles fatigue. Med Sci Sports Exerc 2002;34:1907-12.

35. Yeom CH, Kim TH. Effects of induced fatigue of ankle joint muscle on the capability and recovery of postural control during single-leg stance. KJSB 2012;22:219-28.

36. Goats GC. Massage-the scientific basis of an ancient art. Physiological and therapeutic effects. Br J Sports Med 1994;28:153-6. 